\title{
ON A CLASS OF MARKOV PROCESSES TAKING VALUES ON LINES AND THE CENTRAL LIMIT THEOREM
}

\author{
MASATOSHI FUKUSHIMA and MASUYUKI HITSUDA
}

To Professor Kiyoshi Noshiro on the occasion of his 60-th birthday

\section{§1. Introduction}

We shall consider a class of Markov processes $(n(t), x(t))$ with the continuous time parameter $t \in[0, \infty)$, whose state space is $\{1,2, \ldots, N\} \times R^{1}$. We shall assume that the processes are spacially homogeneous with respect to $x \in R^{1}$. In detail, our assumption is that the transition function

$$
F_{i j}(x, t)=P(n(t)=j, x(t) \leqq x \mid n(0)=i, x(0)=0), \quad t>0,1 \leqq i, j \leqq N, x \in R^{1},
$$

satisfies following conditions $(1,1) \sim(1,4)$.

$(1,1) F_{i j}(x, t)$ is non-negative, and, for fixed $i, j$ and $t$, it is monotone nondecreasing and right continuous in $x \in R^{1}$.

$$
\begin{array}{ll}
(1,2) \quad & F_{i j}(+\infty, t)=\lim _{x \rightarrow \infty} F_{i j}(x, t) \leqq 1, \\
& F_{i j}(-\infty, t)=\lim _{x \rightarrow \infty} F_{i j}(x, t)=0, \quad 1 \leqq i, j \leqq N, \quad t>0, \\
& \sum_{i=1}^{N} F_{i j}(+\infty, t)=1, \quad i=1,2, \ldots, N, \quad t>0, \\
(1,3) \quad & F_{i j}(x, t)=\sum_{k=1}^{N} \int_{R^{1}} F_{i k}(x-y, t) d F_{k j}(y, s) \quad t, s>0, \\
(1,4) \quad 1 \leqq i, k \leqq N, & x \in R^{1}, \\
& \lim _{t \downarrow 0} F_{i j}(x, t)= \begin{cases}\delta_{i j}, & x \in[0,+\infty) \\
0, & x \in(-\infty, 0) .\end{cases}
\end{array}
$$

The central limit theorem for processes of this type, in case of the discrete time parameter and in a special case of the continous time parameter, has been obtained by Keilson and Wishart [3]. In this paper, through introducing a system of generators of the semi-groups related to the processes, we show that the central limit theorem is valid for our cases of the continous time parameter.

Received August 9, 1966. 
With the transition function $F_{i j}(x, t)$ satisfying $(1,1) \sim(1,4)$ we associate its Fourier transform:

$$
f_{i j}(z, t)=\int_{R^{1}} e^{i x z} d F_{i j}(x, t), \quad t>0, \quad 1 \leqq i, j \leqq N, z \in R^{1} .
$$

Then the matrix $\boldsymbol{f}(z, t)=\left(f_{i j}(z, t)\right)$ satisfies the followings:

$$
\begin{aligned}
& (1,5) \quad \sum_{j=1}^{N} f_{i j}(0, t)=1, \quad 1 \leqq i \leqq N, \quad t>0, \\
& (1,6) \quad \boldsymbol{f}(z, t+s)=\boldsymbol{f}(z, t) \boldsymbol{f}(z, s) \quad t, s>0,
\end{aligned}
$$

$(1,7) \quad \boldsymbol{f}(z, t)$ converges, as $t$ tends to zero, to the identity matrix $\boldsymbol{E}$ uniformly in $z \in R^{1}$ in the wide sense, i.e. each element $f_{i j}(z, t)$ of $\boldsymbol{f}(z, t)$ converges to $\delta_{i j}$ uniformly on any compact $z$-set.

In $\S 2$, we shall determine the generator $A(z), z \in R^{1}$, of the semigroups $f(z, t), z \in R^{1}$, (Theorem 1). In particular, if $N=1$, our expression of $\boldsymbol{A}(z)$ is no other than the so-called Lévy-Khintchine formula. $\left\{\boldsymbol{A}(z), z \in R^{1}\right\}$ in Theorem 1 characterizes all the processes whose transition functions satisfy $(1,1) \sim(1,4)$.

$\S 3$ will be devoted to the proof of the central limit theorem for our processes under some assumptions placed upon $\boldsymbol{A}(z)$ (Theorem 2). Our procedure in this section is essentially due to Keilson and Wishart [3].

In $\$ 4, m$ and $v$ which are defiend by the first and second derivatives respectively of an eigen value $\lambda(z)$ of $\boldsymbol{A}(z)$ will be expressed explicitly by $\boldsymbol{A}(z)$ and its eigenvectors. We shall further solve affirmatively the Harris' conjecture related to the expectation processes of the electron-photon cascade (Harris [2], page 194).

\section{§2. The generators of semigroups $f(z, t)$.}

Throughout $\S 2, \S 3$ and the first half of $\S 4$, we shall assume that we are given a transition function $F_{i j}(x, t)$ on $\{1,2, \ldots, N\} \times R^{1}$ satisfiying $(1,1) \sim(1,4)$.

In this section, we shall show the existence of the generator

$$
\boldsymbol{A}(z)=\lim _{t \downarrow 0} \frac{\boldsymbol{f}(z, t)-\boldsymbol{E}}{t}(\text { Lemma } 1),
$$

and determine all the possible types of the generator $\boldsymbol{A}(z)$ (Theorem 1).

Lemma 1. The limit

$$
\lim _{t \downarrow 0} \frac{\boldsymbol{f}(z, t)-\boldsymbol{E}}{t}=\boldsymbol{A}(z)
$$


ON A CLASS OF MARKOV PROCESSES TAKING VALUES ON LINES AND THE CENTRAL LIMIT THEOREM 49

exists and the convergence is uniformly in $z \in R^{1}$ in the wide sence.

Proof. Let us fix $a>0$ arbitrarily. From the property $(1,7)$, we can choose $v>0$ such that, for $z \in[-a, a]$, the matrix

$$
\boldsymbol{\Lambda}_{v}(z)=\int_{0}^{v} \boldsymbol{f}(z, s) d s=\left(\int_{0}^{v} f_{i j}(z, s) d s\right)
$$

has its inverse $\boldsymbol{\Lambda}_{v}^{-1}(z)$. Since

$$
\boldsymbol{\Lambda}_{v}(z) \boldsymbol{f}(z, t)=\int_{0}^{v} \boldsymbol{f}(z, s) \boldsymbol{f}(z, t) d s=\int_{0}^{v} \boldsymbol{f}(z, s+t) d s=\int_{t}^{v+t} \boldsymbol{f}(z, s) d s,
$$

we have

$$
\boldsymbol{f}(z, t)=\boldsymbol{\Lambda}_{v}^{-1}(z) \int_{t}^{v+t} \boldsymbol{f}(z, s) d s
$$

Therefore we can obtain that

$$
\begin{aligned}
& \lim _{h \downarrow 0} \frac{\boldsymbol{f}(z, h)-\boldsymbol{E}}{h}=\lim _{h \downarrow 0} \boldsymbol{\Lambda}_{v}^{-1}(z) \frac{1}{h}\left[\int_{h}^{v+h} \boldsymbol{f}(z, s) d s-\int_{0}^{v} \boldsymbol{f}(z, s) d s\right] \\
= & \boldsymbol{\Lambda}_{v}^{-1}(z) \lim _{h \downarrow 0} \frac{1}{h}\left[\int_{v}^{v+h} \boldsymbol{f}(z, s) d s-\int_{0}^{h} \boldsymbol{f}(z, s) d s\right] \\
= & \boldsymbol{\Lambda}_{v}^{-1}(z)[\boldsymbol{f}(z, v)-E], \quad z \in[-a, a] .
\end{aligned}
$$

The convergence in question is uniform since $\boldsymbol{f}(z, s)$ converges to $\boldsymbol{f}(z, v)$ uniformly in $z \in[-a, a]$ as $s$ tends to $v$. The right side of the above equation should be independent of $v$ because the left does not depend on $v$.

Thus we have proved Lamma 1.

Remark. We can express $\boldsymbol{f}(z, t)$ in the form $\exp \{\boldsymbol{A}(z) t\}$. In fact, $\boldsymbol{f}(z, t)$ is the unique solution of the equation

$$
\frac{\partial}{\partial t} \boldsymbol{f}(z, t)=\boldsymbol{A}(z) \boldsymbol{f}(z, t)
$$

with the initial condition

$$
\lim _{t \downarrow 0} \boldsymbol{f}(z, t)=E
$$

The following theorem gives us the possible types of $\boldsymbol{A}(z)$.

Theorem 1. The elements $a_{i j}(z), i, j=1,2, \ldots, N$, of $\boldsymbol{A}(z)$ can be expressed in the form, 


$$
\begin{aligned}
a_{i j}(z)= & \int_{-\infty}^{\infty} e^{i z x} \Gamma_{i j}(d x) \quad i \neq j, \quad 1 \leqq i, j \leqq N, \\
a_{i i}(z)= & -\sum_{j \neq i} \Gamma_{i j}\left(R^{1}\right)+i \nu_{i} z-\frac{\sigma_{i}{ }^{2}}{2} z^{2}+\int_{|u|>1}\left(e^{i z v}-1\right) \Pi_{i}(d u) \\
& +\int_{|u| \leqq 1}\left(e^{i z u}-1-i z u\right) \Pi_{i}(d u), \quad i=1,2, \ldots, N
\end{aligned}
$$

where $\Gamma_{i j}{ }^{\prime} s, 1 \leqq i \neq j \leqq N$, are finite measures on $R^{1}, \nu_{i}{ }^{\prime} s, i=1, \ldots, N$ are real numbers, $\sigma_{i}$ 's, $i=1, \ldots, N$ are real and positive, and $\Pi_{i}$ 's, $i=1, \ldots, N$ are measures on $R^{1}$ such that $\int_{|u| \leqq 1} x^{2} \Pi_{i}(d x)<\infty$ and $\Pi_{i}(u ;|u|>\varepsilon)<\infty$ for any $\varepsilon>0$.

Proof. In case that $i \neq j$, we can see by Lemma 1 that the convergence

$$
a_{i j}(z)=\lim _{h \downarrow 0} \frac{f_{i j}(z, h)}{h}
$$

is uniform in the wide sence in $z \in R^{1}$, and therefore $a_{i j}(z)$ is a positive definite function of $z$ because $\frac{f_{i j}(z, h)}{h}$ has the same property. Then there exists a finite measure $\Gamma_{i j}$ such that

$$
a_{i j}(z)=\int_{-\infty}^{\infty} e^{i z x} \Gamma_{i j}(d x)
$$

In case that $i=j$, we note that

$$
\frac{f_{i i}(z, h)-1}{h}=\frac{e^{a_{i i}(z) h}-e^{a_{i i}(0) h}}{h}+\frac{e^{a_{i i}(0) h}-1}{h}+\frac{f_{i i}(z, h)-e^{a_{i i}(z) h}}{h} .
$$

Then, $\frac{f_{i i}(z, h)-e^{a_{i i}(z) h}}{h}$ tends to zero as $h \downarrow 0$ by virtue of the remark after Lemma 1. If we remark that $a_{i i}(0)=-\sum_{j=i}^{N} a_{i j}(0)=-\sum_{j=i}^{N} \Gamma_{i j}\left(R^{1}\right), \frac{e^{a_{i i}(0)}-1}{h}$ tends to $-\sum_{j=i}^{N} \Gamma_{i j}\left(R^{1}\right)$ as $h \downarrow 0$. The limit of $\frac{e^{a_{i i}(z) h}-e^{a_{i i}(0) h}}{h}=e^{a_{i i}(0) h} \cdot \frac{e^{\left(a_{i i}(z)-a_{i i}(0)\right) h}-1}{h}$ as $h \downarrow 0$ is the Lévy-Khintchine formula:

$$
i \nu_{i} z-\frac{\sigma_{i}^{2}}{2} z^{2}+\int_{|u|>1}\left(e^{i z u}-1\right) \Pi_{i}(d u)+\int_{|u| \leqq 1}\left(e^{i z u}-1-i z u\right) \Pi_{i}(d u)
$$

(see for example Gnedenko and Kolmogorov [1]).

Remark: From the above discussion, we can see that $\Gamma_{i j}, \nu_{i}, \sigma_{i}$; and $\Pi_{i}$ are uniquely determined by the transition function $F_{i j}(x, t)$ satisfying $(1,1) \sim$ $(1,4)$. 


\section{§3. The central limit theorem.}

Let $\boldsymbol{A}(z)$ be the matrix defined in Lemma 1. We shall assume Assumption 1.

$$
\begin{aligned}
& \int_{-\infty}^{\infty} x^{2} \Gamma_{i j}(d x)<+\infty, \quad i \neq j, 1 \leqq i, j \leqq N, \\
& \int_{-\infty}^{\infty} x^{2} \Pi_{i}(d x)<+\infty, \quad 1 \leqq i \leqq N .
\end{aligned}
$$

Assumption 2. $\boldsymbol{A}(0)$ is irreducible.

The Assumption 1 is equivalent to the fact that

$\left(^{*}\right) \quad \int_{-\infty}^{\infty} x^{2} d F_{i j}(x, 1)<\infty \quad$ for any $i, j=1, \ldots, N$.

In fact, Assumption 1 is equivalent to that

(**) $\boldsymbol{A}(z)$ is twice differentiable with respect to $z$.

And (**) is equivalent to the same property of $e^{A(z)}=f(z, 1)$. This is no other than (*) (see Feller [4] page 485).

By virtue of Assumption 2, $\boldsymbol{f}(0,1)=e^{\boldsymbol{A}(0)}$ is a positive stochastic matrix and therefore, by Perron-Frobenius' theorem, it has the simple eigenvalue 1 and the absolute values of the other $\mathrm{N}-1$ eigenvalues are less than 1 . Therefore, the equation

$$
\operatorname{det}(\boldsymbol{A}(0)-\lambda \boldsymbol{E})=\pi(0, \lambda)=0
$$

has a simple root $\lambda=0$, and all the other roots have negative real parts. From these facts we can derive the next Lemma.

Lemma 2. There is a function $\lambda(z)$ defined on some neighbourhood $(-a, a)$ of $z=0$ which has the continuous first and second derivatives in $(-a, a)$ and satisfies that

$$
\begin{aligned}
& \pi(z, \lambda(z))=0, \quad z \in(-a, a) \\
& \lambda(0)=0 \\
& \text { where } \pi(z, \lambda)=\operatorname{det}(\boldsymbol{A}(z)-\lambda \boldsymbol{E}) .
\end{aligned}
$$

Further, $\lambda^{\prime}(0)$ is purely imaginary and $\lambda^{\prime \prime}(0)$ is real and non-positive.

Proof. As $\lambda=0$ is a simple root of the equation $\Pi(0, \lambda)=0$, we know that

$$
\begin{gathered}
\pi_{\lambda}(0,0)=\frac{\partial \pi}{\partial \lambda}(0,0) \neq 0 . \text { If we put } \\
\pi(z, \lambda)=\pi_{1}\left(z, \lambda_{1}, \lambda_{2}\right)+i \pi_{2}\left(z, \lambda_{1}, \lambda_{2}\right)
\end{gathered}
$$


where $\pi_{i}\left(z, \lambda_{1}, \lambda_{2}\right), i=1,2$, are real valued functions and $\lambda_{1}=\Re e \lambda, \lambda_{2}=\Im \mathfrak{m} \lambda$, then we have

$$
\pi_{1}(0,0,0)=0, \quad \pi_{2}(0,0,0)=0 .
$$

On the otherhand, $\pi(z, \lambda)$ is analytic in $\lambda$. Therefore we have by CauchyRiemann's equation that

$$
\begin{aligned}
& \left|\begin{array}{ll}
\frac{\partial \pi_{1}}{\partial \lambda_{1}}(0,0,0) & \frac{\partial \pi_{2}}{\partial \lambda_{1}}(0,0,0) \\
\frac{\partial \pi_{1}}{\partial \lambda_{2}}(0,0,0) & \frac{\partial \pi_{2}}{\partial \lambda_{2}}(0,0,0)
\end{array}\right|=\left|\begin{array}{cc}
\frac{\partial \pi_{1}}{\partial \lambda_{1}}(0,0,0) & \frac{\partial \pi_{2}}{\partial \lambda_{1}}(0,0,0) \\
-\frac{\partial \pi_{2}}{\partial \lambda_{1}}(0,0,0) & \frac{\partial \pi_{1}}{\partial \lambda_{1}}(0,0,0)
\end{array}\right| \\
& =\left(\frac{\partial \pi_{1}}{\partial \lambda_{1}}(0,0,0)\right)^{2}+\left(\frac{\partial \pi_{2}}{\partial \lambda_{1}}(0,0,0)\right)^{2} \neq 0 .
\end{aligned}
$$

Now the first part of Lemma 2 is the direct consequence of the implicit function theorem.

Now, it is easy to see by the form of $\boldsymbol{A}(z)$ that $\pi_{z}(0,0)=\frac{\partial}{\partial z} \pi(0,0)$ is pure imaginary and $\pi_{\lambda}(0,0)$ is real. Therefore $\lambda^{\prime}(0)=\frac{-\pi_{z}(0,0)}{\pi_{\lambda}(0,0)}$ is pure imaginary. By the same method we can see that $\lambda^{\prime \prime}(0)$ is real. Let us note that $\sum_{i=1}^{N}\left|f_{i j}(z, 1)\right| \leqq 1$ where $f_{i j}(z, 1)$ is $(i, j)$-element of the matrix $e^{\boldsymbol{A}(z)}=\boldsymbol{f}(z, 1)$. This implies, applying Frobenius' theorem again, all the eigenvalues of $\boldsymbol{A}(\boldsymbol{z})$ have non-positive real parts. Especially, $\Re e \lambda(z) \leqq 0$ and $\Re e \lambda(z)$ attains its maximum 0 at $z=0$. Thus

$$
\left.\frac{d^{2}}{d z} \Re \mathrm{e} \lambda(z)\right]_{z=0}=\Re \mathrm{Re} \lambda^{\prime \prime}(0)=\lambda^{\prime \prime}(0) \leqq 0 .
$$

Remark. We may assume without loss of generality that for $z \in(-a, a)$ $\lambda(z)$ is the eigenvalue of $\boldsymbol{A}(z)$ the real part of which is greater than the real parts of any other eigenvalues, because the eigenvalues of $\boldsymbol{A}(\boldsymbol{z})$ are continuous in $z$, and the eigenvalue $\lambda(0)=0$ of $\boldsymbol{A}(0)$ has the maximum real part. In the following we assume the above property of $\lambda(z)$ in $z \in(-a, a)$.

Consider a probality measure $\mu=\left(\mu_{1}, \mu_{2}, \ldots \ldots, \mu_{N}\right)$ on $\{1,2, \ldots \ldots, N\} \times R^{1}$, where $\mu_{i}^{\prime}$ s are measues on $R^{1}$ such that $\sum_{i=1}^{N} \mu_{i}\left(R^{1}\right)=1$. Let us put

$$
F_{j}^{\mu}(x, t)=\sum_{i=1}^{N} \int_{-\infty}^{\infty} \mu_{i}(d y) F_{\imath j}(x-y, t), \quad 1 \leqq j \leqq N, \quad \text { and }
$$




$$
\boldsymbol{F}^{\mu}(x, t)=\left\{F_{1}^{\mu}(x, t), F_{2}^{\mu}(x, t), \ldots \ldots, F_{N}^{\mu}(x, t)\right\}
$$

Then $\boldsymbol{F}^{\mu}(x, t)$ determines the distribution of a Markov process on $\{1,2, \ldots \ldots, N\}$ $\times R^{1}$ at time $t$ with the initial distribution $\mu$. We further put

$$
\boldsymbol{f}^{\mu}(z, t)=\left\{\int_{-\infty}^{\infty} e^{i x z} d F_{1}^{\mu}(x, t), \int_{-\infty}^{\infty} e^{i x z} d F_{2}^{\mu}(x, t), \ldots \ldots, \int_{-\infty}^{\infty} e^{i x z} d F_{N}^{\mu}(x, t)\right\} .
$$

Then,

$$
\boldsymbol{f}^{\mu}(z, t)=\boldsymbol{f}^{\mu}(z, 0+) \boldsymbol{f}(z, t)
$$

where $\boldsymbol{f}^{\mu}(z, 0+)$ is the Fourier transform of $\mu$. Define $m$ and $v$ by the formula

$$
m=-i \lambda^{\prime}(0), \quad v=-\lambda^{\prime \prime}(0) \text {. }
$$

TheOREm 2. For any initial distribution $\mu$ on $\{1,2, \ldots \ldots, N\} \times R^{1}$,

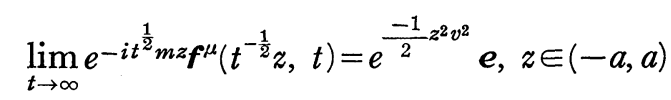

holds, where $\boldsymbol{e}=\left(e_{1}, e_{2}, \ldots \ldots, e_{N}\right)$ is the left eigenvector belonging to $\boldsymbol{A}(0)$ for the eigenvalue 0 , and $\sum_{i=1}^{N} e_{i}=1$.

Proof. There is a regulaer matrix $\boldsymbol{T}(z)$ for $z \in(-a, a)$ such that

$$
\boldsymbol{A}(z)=\boldsymbol{T}^{-1}(z) \boldsymbol{J}(z) \boldsymbol{T}(z)
$$

where $\boldsymbol{J}(z)$ is a matrix of the Jordan's normal form whose $(1,1)$-element is $\lambda(z)$. Here we can choose $\boldsymbol{T}(z)$ to be continuous in $z \in(-a, a)$. Now we have

$$
\boldsymbol{f}^{\mu}\left(t^{-\frac{1}{2}} z, t\right) e^{-i z m t^{\frac{1}{2}}}=\boldsymbol{f}^{\mu}\left(t^{-\frac{1}{2}} z, 0\right) \boldsymbol{T}^{-1}\left(t^{-\frac{1}{2}} z\right) e^{-i z m t^{-\frac{1}{2}}} \exp \left(t \boldsymbol{J}\left(t^{-\frac{1}{2}}\right)\right) \boldsymbol{T}\left(t^{-\frac{1}{2}} z\right) .
$$

In order to investigate the limit of the above expression, we first note the following

$$
\lim _{t \rightarrow \infty} \boldsymbol{f}^{\mu}\left(t^{-\frac{1}{2}} z, 0\right)=\lim _{z \rightarrow 0} \boldsymbol{f}^{\mu}(z, 0)=\boldsymbol{f}^{\mu}(0,0) .
$$

Since the first column $\boldsymbol{e}(z)$ of $\boldsymbol{T}(z)$ is the left eigenvector and the first row $\boldsymbol{i}(z)$ of $\boldsymbol{T}(z)$ is the right eigenvector of $\boldsymbol{A}(z)$ belonging to the eigenvalue $\lambda(z)$, we may assume that $\boldsymbol{e}(0)$ and $\boldsymbol{i}(0)$ are of the form

$$
\begin{aligned}
\boldsymbol{e}(0) & =\boldsymbol{e}=\left(e_{1}, e_{2}, \ldots \ldots, e_{N}\right) \\
\boldsymbol{i}(0) & =\left(\begin{array}{c}
1 \\
1 \\
\vdots \\
1
\end{array}\right) .
\end{aligned}
$$


By the continuity of each element of $\boldsymbol{T}(z)$, we have

$$
\begin{aligned}
& \lim _{t \rightarrow \infty} T\left(t^{\frac{-1}{2}} z\right)=\lim _{z \rightarrow 0} T(z)=\left(\begin{array}{c}
e_{1}, e_{2}, \ldots . ., e_{N} \\
*
\end{array}\right) \\
& \lim _{t \rightarrow \infty} T^{-1}\left(t^{\frac{-1}{2}} z\right)=\lim _{z \rightarrow 0} T^{-1}(z)=\left(\begin{array}{ccc}
1 & \\
1 & * \\
\vdots &
\end{array}\right)
\end{aligned}
$$

The absolute values of the $(i, j)$-elements of $\exp \{t \boldsymbol{J}(z)\}$ for $i, j \geqq 2$ and $j-i=l \geqq 0$ are less than $\frac{1}{l !} t e^{-\alpha(z) t}$, where $-\alpha(z)$ denotes the maximum real part of the eigenvalues of $\boldsymbol{A}(z)$ except $\lambda(z)$.

On the other hand the $(i, j)$-elements of $\exp \{t \boldsymbol{J}(z)\}$ for $j-i<0$ or for $i=1$, $j \neq i$ are equal to 0 . Since $\sup _{z \in(-a, a)} e^{-\alpha(z)}<1$, the $(i, j)$-elements of $\exp \{t \boldsymbol{J}(z)\}$ for $(i . j) \neq(1,1)$ converge to 0 as $t \rightarrow \infty$. For the $(1,1)$-element, we get

$$
\lim _{t \rightarrow \infty} e^{-i z m t^{\frac{1}{2}}} e^{t \lambda\left(t^{\frac{-1}{2}} z\right)}=e^{\frac{-1}{2} z^{2} v^{2}},
$$

with $m$ and $v$ defined by $(3,1)$. Therefore

$$
\lim _{t \rightarrow \infty} e^{-i z m t^{\frac{1}{2}}} \exp \left\{t \boldsymbol{J}\left(t^{\frac{-1}{2}} z\right)\right\}=e^{\frac{-1}{2} z^{2} v^{2}}\left(\begin{array}{c:c}
1 & 0 \ldots \ldots 0 \\
\hdashline 0 & \\
\vdots & 0 \\
0 &
\end{array}\right)
$$

holds. Consequently we get $(3,1)$ by $(3,3) \sim(3,7)$ and the proof is complete.

Corollary. In case $v=-\lambda^{\prime \prime}(0)>0$, we have

$$
\lim _{t \rightarrow \infty} \boldsymbol{F}^{\mu}\left(x t^{\frac{1}{2}}+t m, t\right)=\int_{0}^{\frac{x}{v}} \frac{e^{-\frac{y^{2}}{2}}}{\sqrt{2 \pi}} d y \cdot e
$$

for any initial distribution $\mu$.

\$4. The expression of $m$ and $v$, and the expectation process of the eletron-photon cascade.

Let $\boldsymbol{e}(z)$ and $\boldsymbol{i}(z)$ be the left and right eigenvector of $\boldsymbol{A}(z)$ belonging to $\lambda(z)$ respectively, as in the proof of Theorem 2. We can assume that they are twice differentiable in the neighbourhood of the origin and $\boldsymbol{e}(0)=\boldsymbol{e}, \boldsymbol{i}(0)=\mathbf{1}$.

Since $\lambda(0)=0$, taking dreivatives of the equations 


$$
\begin{aligned}
& \boldsymbol{e}(z) \boldsymbol{A}(z)=\lambda(z) \boldsymbol{e}(z) \quad \text { and } \\
& \boldsymbol{A}(z) \boldsymbol{i}(z)=\lambda(z) \boldsymbol{i}(z)
\end{aligned}
$$

we have

$$
\boldsymbol{e}^{\prime}(0) \boldsymbol{A}(0)+\boldsymbol{e} \boldsymbol{A}^{\prime}(0)=\lambda^{\prime}(0) \boldsymbol{e} \quad \text { and }
$$

$$
\boldsymbol{A}(0) \boldsymbol{i}^{\prime}(0)+\boldsymbol{A}^{\prime}(0) \mathbf{1}=\lambda^{\prime}(0) \mathbf{1} \text {. }
$$

By $(4,3)$, we have

$$
m=-i \lambda^{\prime}(0)=-\boldsymbol{e} \boldsymbol{A}^{\prime}(0) \mathbf{1} .
$$

Differentiating $(4,1)$ twice and noting $(4,4)$, we have

$$
v=-\lambda^{\prime \prime}(0)=-\boldsymbol{e} \boldsymbol{A}^{\prime \prime}(0) \mathbf{1}+2 \boldsymbol{e}^{\prime}(0) \boldsymbol{A}(0) \boldsymbol{i}^{\prime}(0) .
$$

The following Lemma 3 will illustrate the probalibistic measning of $m$ and $v$. Let $\{n(t), x(t)\}$ be the Markov process on $\{1,2, \ldots \ldots, N\} \times R^{1}$ whose transition function are governed by $F_{i j}(x, t)$ and whose initial distrivution is $\delta_{0}(d x) e$, where $\delta_{0}$ is the $\delta$-measure concentrated at the origin.

LEMMA 3. $\quad m=-i \boldsymbol{e} \boldsymbol{A}^{\prime}(\boldsymbol{0}) \mathbf{1}=\frac{\boldsymbol{E}(x(t))}{t}$, for any $t>0$

$$
v=-\boldsymbol{e} \boldsymbol{A}^{\prime \prime}(0) \mathbf{1}+2 \boldsymbol{e}^{\prime}(0) \boldsymbol{A}(0) \dot{\boldsymbol{i}}^{\prime}(0)=\lim _{t \rightarrow+\infty} \frac{\boldsymbol{E}[x(t)-\boldsymbol{E}(x(t))]^{2}}{t} .
$$

Proof. We can see that

$$
\boldsymbol{E}(x(t))=-i \boldsymbol{e}\left(\frac{\partial}{\partial z} \boldsymbol{f}(z, t)\right) \mathbf{1}=-i \boldsymbol{e}\left(\frac{\partial}{\partial z} e^{t \mathbf{A}(z)}\right) \mathbf{1}=-i \text { te } A(0) \mathbf{1} m t,
$$

and

$$
\begin{array}{r}
\boldsymbol{E}[x(t)-\boldsymbol{E}(x(t))]^{2}=\boldsymbol{E}\left(x(t)^{2}\right)-m^{2} t^{2}=-\boldsymbol{e}\left(\frac{\partial^{2}}{\partial z^{2}} e^{t} \boldsymbol{A}(\boldsymbol{z})\right) \\
\mathbf{1}+\lambda^{\prime}(0)^{2} t^{2} \\
=-t \boldsymbol{e} \boldsymbol{A}^{\prime \prime}(0) \mathbf{1}-2 \sum_{k=2}^{\infty} \frac{t^{k}}{k !} \boldsymbol{e} \boldsymbol{A}^{\prime}(0) \boldsymbol{A}^{k-2}(0) \boldsymbol{A}^{\prime}(0) \mathbf{1}+\lambda^{\prime}(0)^{2} t^{2} .
\end{array}
$$

While, the second term of the last expression is, by virtue of $(4,3)$ and $(4,4)$, equal to $-\lambda^{\prime}(0)^{2} t^{2}-2 \boldsymbol{e}^{\prime}(0)(\exp (t \boldsymbol{A}(0))-\boldsymbol{E}-t \boldsymbol{A}(0)) \boldsymbol{l}^{\prime}(0)$. Thus, we see that the second equation of Lemma 3 is valid.

In the remainder of this section, let us discuss the case where $N=2$. In this case, $\boldsymbol{A}(0)$ can be written in the form $\boldsymbol{A}(0)=\left(\begin{array}{rr}-a & a \\ b-b\end{array}\right)$ and by the assumption that $\boldsymbol{A}(0)$ is irreducible, $a>0$ and $b>0$ hold. Therefore 


$$
\boldsymbol{A}(0)^{n}=(-1)^{n-1}(a+b)^{n-1} \boldsymbol{A}(0), n>1,
$$

and, by $(4,3),(4,4)$ and $(4,6)$, we have

$$
(4,7) \quad v=-\boldsymbol{e} \boldsymbol{A}^{\prime \prime}(0) \mathbf{1}+\frac{2}{(a+b)^{2}} \boldsymbol{e} \boldsymbol{A}^{\prime}(0) \boldsymbol{A}(0) \boldsymbol{A}^{\prime}(0) \mathbf{1},
$$

The expectation process introduced by Harris ([2] Chap. VII) related to the electron-photon cascade can be dealt with as a special case of our discussion with $N=2$.

Harris has conjectured that the central limit theorem holds for the expectation process, with $m$ and $v$ given by the right hand sides of the equations $(4,5)$ and $(4,7)$ respectively ([2] page 198). Theorem 2 and the above discussions show the validity of his conjecture.

\section{REFERENCES}

[1] B.V. Gnedenko and A.N. Kolmogorov: Limit distributions for sums of independent random variables, Addison-Wesley Pub. Comp., (1954), (Translated from the Russian.)

[2] T.E. Harris: The theory of branching processes, Springer-Verlag, (1963).

[3] J. Keilson and D.M.G. Wishart: A central limit theorem for processes detined on a finite Markov chain, Proc. Camb. Phil. Soc., 60 (1964), 547-567.

[4] W. Feller: An introduction to probability theory and its application vol. 2, John Wiley \& Sons, (1966).

Tokyo University of Education and Nagoya University 